\section{REVISTA INTERNACIONAL DE CIENCIAS DEL DEPORTE International Journal of Sport Science}

doi:10.5232/ricyde2009.01501

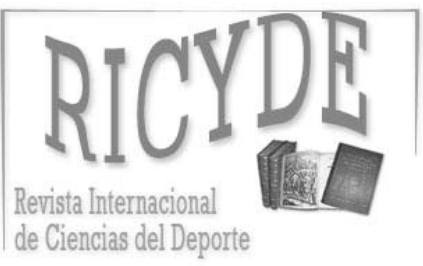

International Journal of Sport Science VOLUMEN V - AÑO V

Páginas:1-16 ISSN:1885-3137

No 15 - Abril - 2009

Rev. int. cienc. deporte

\title{
Perfil antropométrico de las jugadoras de baloncesto españolas. Análisis en función del nivel competitivo y de la posición específica de juego.
}

\section{Anthropometric profile of Spanish female basketball players. Analysis by level and by playing position.}

\author{
Irene Salgado Sánchez; Silvia Sedano Campo; Ana de Benito Trigueros; \\ José María Izquierdo Velasco; Gonzalo Cuadrado Sáenz \\ Facultad de Ciencias de la Actividad Física y del Deporte \\ Universidad de León
}

\section{Resumen}

El objetivo del estudio es determinar el perfil antropométrico de las jugadoras de baloncesto en España en función del nivel competitivo y del puesto específico de juego. La muestra se compone de 64 jugadoras de baloncesto pertenecientes a equipos federados de la Liga Femenina de Baloncesto $(n=20)$, Liga Femenina 2 de Baloncesto $(n=22)$ y Primera Nacional $(n=22)$.

En total se registraron 16 medidas antropométricas (masa corporal, talla, siete pliegues, tres diámetros y cuatro perímetros) para posteriormente determinar la composición corporal y el somatotipo. En términos generales se observan diferencias tanto en composición corporal como en somatotipo entre diferentes niveles competitivos, diferencias que pueden considerarse favorables a las jugadoras de mayor nivel en casi todas las variables examinadas. Podemos hablar de la existencia de un perfil cineantropométrico específico en función de la posición de juego. Las pívots son las jugadoras más altas y pesadas, y con mayor porcentaje de grasa, seguidas por las aleros y por último las bases.

Palabras clave: baloncesto femenino, antropometría, composición corporal, somatotipo, somatocarta.

\begin{abstract}
The purpose of this study was to identify the anthropometric profile of Spanish female basketball players by level and by playing position. The sample was formed by 64 female baskeball players who practiced basketball in collegiated teams of Liga Femenina de Baloncesto (20), Liga Femenina 2 de Baloncesto (22) and Primera Nacional (22).

Sixteen body dimensions were measured (body mass, height, seven skinfolds, three diameters and four girths) in order to determine body composition and somatotype. The results of the study demonstrated that body composition and somatotype were different between levels of play. Both data were favourable to the highest level in almost all the examined variables. When we analysed body composition and somatotype by playing position we realised that an specific anthropometric profile existed in all playing positions. Center were taller and heavier than forwards and guards and also had higher body fat percentages than the other groups.
\end{abstract}

Key words: female basketball, anthropometry, body composition, somatotype, somatochart. 
Salgado, I.; Sedano, S.; de Benito, A.; Izquierdo, J.M.; Cuadrado, G. (2009). Perfil antropométrico de las jugadoras de baloncesto españolas. Análisis en función del nivel competitivo y de la posición específica de juego. Revista Internacional de Ciencias del Deporte. 15(5), 1-16. http://www.cafyd.com/REVISTA/01501.pdf

\section{Introducción}

- 1 baloncesto es un deporte de equipo aeróbico-anaeróbico alternado con un alto nivel de ـexigencia física, técnica y táctica (Franco y cols., 1998). Conlleva una serie de esfuerzos intermitentes, una alternancia de sprints cortos y de saltos y descansos activos o pasivos (Cometti, 2002). En la misma línea concluye Zaragoza (1996), quien afirma que se trata de un deporte aeróbico-anaeróbico alternado, con fases breves donde se producen acciones máximas. A este respecto, Lorenzo (1998) es muy claro al afirmar que se trata de un deporte de fuerza y velocidad. Y con una mayor concreción, considera que las cualidades físicas predominantes son: velocidad de reacción, capacidad de aceleración, velocidad gestual, fuerza explosiva y resistencia a los esfuerzos máximos (velocidad, fuerza explosiva).

Por otro lado la evolución tanto de las reglas como de la táctica ha dado lugar a la creación de tres posiciones específicas para los jugadores, existiendo unas características físicas y técnicotácticas claramente diferenciadas entre ellas: bases, aleros y pívots (Sallet y cols., 2005). Las jugadoras base tienen un rol fundamental en la organización del juego, encargándose de subir el balón de campo defensivo a campo ofensivo, y ocupando posiciones lejanas a la canasta. Los aleros ayudan al base en la organización del juego y suelen ser los encargados de finalizar los contraataques, siendo jugadores ágiles y muy rápidos. Por último, los pívots ocupan posiciones cercanas al aro usando su superioridad en la talla y la masa corporal para las situaciones de rebote.

Debido a esta división por puestos específicos, las características antropométricas de los hombres jugadores de baloncesto en función de dichas posiciones han suscitado especial atención por parte de numerosos investigadores (Galiano, 1987; Janeira, 1994; Apostolidis y cols., 2003; Sallet y cols., 2005). Sin embargo, el número de estudios relacionados con las características antropométricas en mujeres jugadoras de baloncesto es muy escaso (Häkkinen, 1993; Smith y cols., 1991).

En cualquier caso, la realización de estudios antropométricos en deportes de equipo permite conocer la dirección que toma la forma externa de cada jugador, controlar y evaluar los efectos del entrenamiento en el organismo y conocer las posibles diferencias existentes en función de las posiciones habituales y del nivel competitivo (Liparotti, 2004).

El objetivo del presente estudio es determinar el perfil antropométrico de las jugadoras de baloncesto en España en función del nivel competitivo y del puesto específico de juego.

\section{Material y método}

\section{Muestra}

La muestra se compone de 64 jugadoras de baloncesto, (cuarenta y nueve españolas, cuatro brasileñas, cinco estadounidenses, dos francesas, una israelí, una inglesa, una ecuatoriana y una búlgara) todas ellas pertenecientes a Clubes de Castilla y León, divididas a su vez en tres grupos:

Grupo de Liga Femenina (LFB): 20 mujeres pertenecientes a equipos de la LFB en la temporada 2007-2008, con una edad media de 25,6 \pm 3,9 años, una frecuencia media de entrenamiento de 18,3 $\pm 1,4$ horas a la semana y una frecuencia de competición de carácter semanal. Grupo de Liga Femenina 2 (LF2): 22 mujeres pertenecientes a equipos que 
Salgado, I.; Sedano, S.; de Benito, A.; Izquierdo, J.M.; Cuadrado, G. (2009). Perfil antropométrico de las jugadoras de baloncesto españolas. Análisis en función del nivel competitivo y de la posición específica de juego. Revista Internacional de Ciencias del Deporte. 15(5), 1-16. http://www.cafyd.com/REVISTA/01501.pdf

participaban en la LF2 en la temporada 2007-2008, con una edad media de 24,9 \pm 5,1 años, una frecuencia media de entrenamiento de $12,5 \pm 1,1$ horas a la semana y una frecuencia de competición de carácter semanal. Grupo de Primera Nacional (PN): 22 mujeres pertenecientes a equipos que participaban en la PN de Baloncesto en la temporada 2007-2008, con una edad media de 22,0 \pm 3,3 años, una frecuencia media de entrenamiento de 6,2 $\pm 1,45$ horas a la semana y una frecuencia de competición de carácter semanal.

\section{Material}

- Báscula TANITA BF-666, (0-150 Kg; precisión de 100 gramos).

- Tallímetro Detecto D52, (60-200 cm; precisión de 1 mm).

- Plicómetro Holtain (0-48 mm; precisión de 0,2mm).

- Calibre Lafayette (0-12 cm; precisión de $1 \mathrm{~mm})$.

- Cinta métrica inextensible Holtain (0-100 cm; precisión de 1 mm).

Para el registro y tratamiento de datos se utilizó un ordenador portátil Pentium IV con el sistema operativo Windows XP (Home edition), el editor de texto WinWord 2000, la hoja de cálculo Excel 2000 y el paquete SPSS 14.0 para Windows.

\section{Procedimiento}

Todas las mediciones fueron tomadas durante la temporada competitiva de todos los equipos en el primer trimestre de 2008.

Tras informar a los responsables de los diferentes clubes deportivos acerca de la metodología y objetivos del estudio y solicitar el correspondiente permiso para la realización de las pruebas, se citó a las jugadoras para informarles de las características del estudio y para pedir su consentimiento informado por escrito. Posteriormente y en un espacio convenientemente habilitado para la toma de datos (habitación amplia con temperatura e iluminación adecuadas) un evaluador experimentado realizó las mediciones necesarias para la determinación de la composición corporal y el somatotipo, contando para ello con la colaboración de un ayudante que anotaba las medidas en una ficha antropométrica específicamente diseñada para el estudio.

Siguiendo los protocolos de medidas antropométricas establecidos por la International Society for the Advancement of Kinanthropometry (ISAK) y tras efectuar la adecuada calibración de los instrumentos se tomaron las siguientes medidas: Talla, masa corporal, siete pliegues (tríceps, subescapular, bíceps, suprailíaco, abdominal, muslo anterior, medial de la pierna), tres diámetros (biepicondíleo del húmero, biestiloideo, bicondíleo del fémur) y cuatro perímetros (brazo relajado, brazo contraído y flexionado, medial del muslo, pierna).

El estudio de la composición corporal se realizó a partir de un modelo de cuatro componentes: 1) Porcentaje de grasa calculado a través de la ecuación propuesta por Faulkner (1968) utilizando seis pliegues cutáneos (Tríceps, subescapular, suprailíaco, abdominal, muslo anterior, medial de la pierna); 2) Masa ósea a través de la fórmula de Von Dobeln modificada por Rocha en 1974; 3) Masa residual hallada a partir de las constantes planteadas por Würch en 1974; 4) Masa muscular obtenida mediante la fórmula de Matiegka (1921) (citado por Esparza y cols., 1993) .

Por su parte el somatotipo se calculó mediante el método antropométrico de Heath - Carter, (Carter, 1975) representándolo gráficamente a través del triángulo de Reuleaux, (Carter, 1975). 
Salgado, I.; Sedano, S.; de Benito, A.; Izquierdo, J.M.; Cuadrado, G. (2009). Perfil antropométrico de las jugadoras de baloncesto españolas. Análisis en función del nivel competitivo y de la posición específica de juego. Revista Internacional de Ciencias del Deporte. 15(5), 1-16. http://www.cafyd.com/REVISTA/01501.pdf

\section{Análisis estadístico}

Una vez finalizada la recogida de datos se procedió al tratamiento estadístico de los mismos. Por un lado se extrajeron los estadísticos descriptivos correspondientes (media \pm desviación estándar (D.E), por otro se utilizó el análisis de varianza de un solo factor (ANOVA) con un intervalo de confianza del 95\% para determinar si las diferencias entre un nivel competitivo y otro eran significativas. Para conocer las diferencias existentes según la posición ocupada habitualmente en el terreno de juego también se realizó el estudio estadístico mediante el ANOVA. En ambos casos, cuando se encontraban diferencias significativas entre los grupos se empleó la prueba de contrastes de Scheffé para localizarlas. Para todos los análisis los resultados eran significativos cuando $p<0.05$.

\section{Resultados}

En primer lugar se ofrecen los resultados haciendo una distinción en función del nivel competitivo y a continuación se muestran los resultados en función de la posición específica de juego.

\section{Resultados obtenidos en función del nivel competitivo}

La tabla I muestra los estadísticos descriptivos (media \pm D.E) de las variables de masa corporal, talla, porcentaje de masa grasa, masa muscular y masa ósea. Los resultados obtenidos en la masa residual se han omitido puesto que, al calcularse a través de unas constantes, y al estar hablando en términos de porcentaje los valores son idénticos para los tres grupos.

Se encontraron diferencias estadísticamente significativas entre grupos en la masa corporal, la talla, el porcentaje de grasa, el porcentaje de masa muscular y el porcentaje de masa ósea.

Tabla I: Resultados obtenidos en todas las variables, para todos los grupos.

\begin{tabular}{|l|c|c|c|c|c|}
\hline \multicolumn{1}{|c|}{ Variable } & LFB & LF2 & PN & F & P \\
\hline Masa Corporal (Kg) & $74,3 \pm 8,0$ & $76,4 \pm 11,5$ & $69,1 \pm 7,2$ & 3.393 & .041 \\
\hline Talla (cm) & $183,2 \pm 7,7$ & $180,2 \pm 7,5$ & $174,8 \pm 7,3$ & 5.615 & .006 \\
\hline Porcentaje de grasa & $12,0 \pm 2,1$ & $13,8 \pm 2,4$ & $12,4 \pm 2,0$ & 3.451 & .039 \\
\hline Porcentaje de masa muscular & $43,3 \pm 3,3$ & $44,4 \pm 3,8$ & $45,8 \pm 3,4$ & 18.309 & .000 \\
\hline Porcentaje de masa ósea & $23,8 \pm 1,3$ & $20,9 \pm 3,0$ & $20,9 \pm 2,5$ & 69.322 & .000 \\
\hline
\end{tabular}

Las pruebas de contrastes de Scheffé localizan dichas diferencias con respecto a la masa corporal entre LF2 y PN; en la talla entre LFB y PN; en el porcentaje de grasa entre LFB y LF2; en el porcentaje de masa muscular entre LFB y LF2, entre LF2 y PN y entre LFB y PN; y en el porcentaje de masa ósea entre LFB y LF2 y entre LF y PN. (Figura 1). 
Salgado, I.; Sedano, S.; de Benito, A.; Izquierdo, J.M.; Cuadrado, G. (2009). Perfil antropométrico de las jugadoras de baloncesto españolas. Análisis en función del nivel competitivo y de la posición específica de juego. Revista Internacional de Ciencias del Deporte. 15(5), 1-16. http://www.cafyd.com/REVISTA/01501.pdf

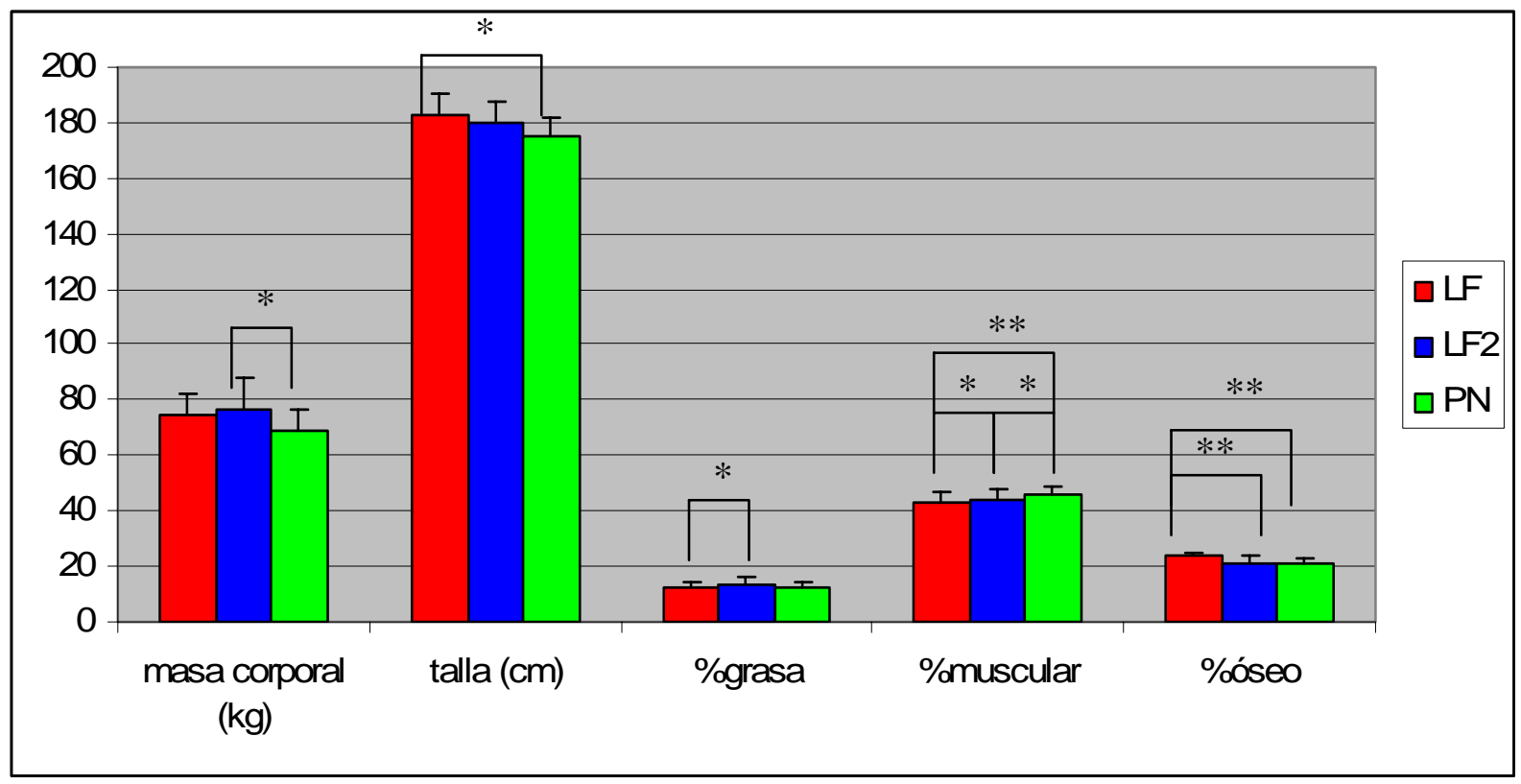

Figura 1. Relación de las diferencias significativas entre categorías. ${ }^{*} \mathrm{p}<0,05 ;{ }^{* *} \mathrm{p}<0,01$. MC masa corporal; T talla; G graso; M muscular; O óseo; R residual.

En la figura 2 se presentan los resultados obtenidos en la distribución de la composición corporal según el modelo de cuatro componentes usado en su cálculo, en función de la categoría.

Con respecto a la masa grasa, son las jugadoras de LF2 las que presentan mayores valores, seguidas de las de PN y LFB respectivamente; en las masa muscular, son las jugadoras de PN las que presentan valores más altos, seguidos de LF2 y LFB respectivamente; el porcentaje de masa ósea más elevado lo presentan las jugadoras de LFB, seguidas por las de PN y LF2.

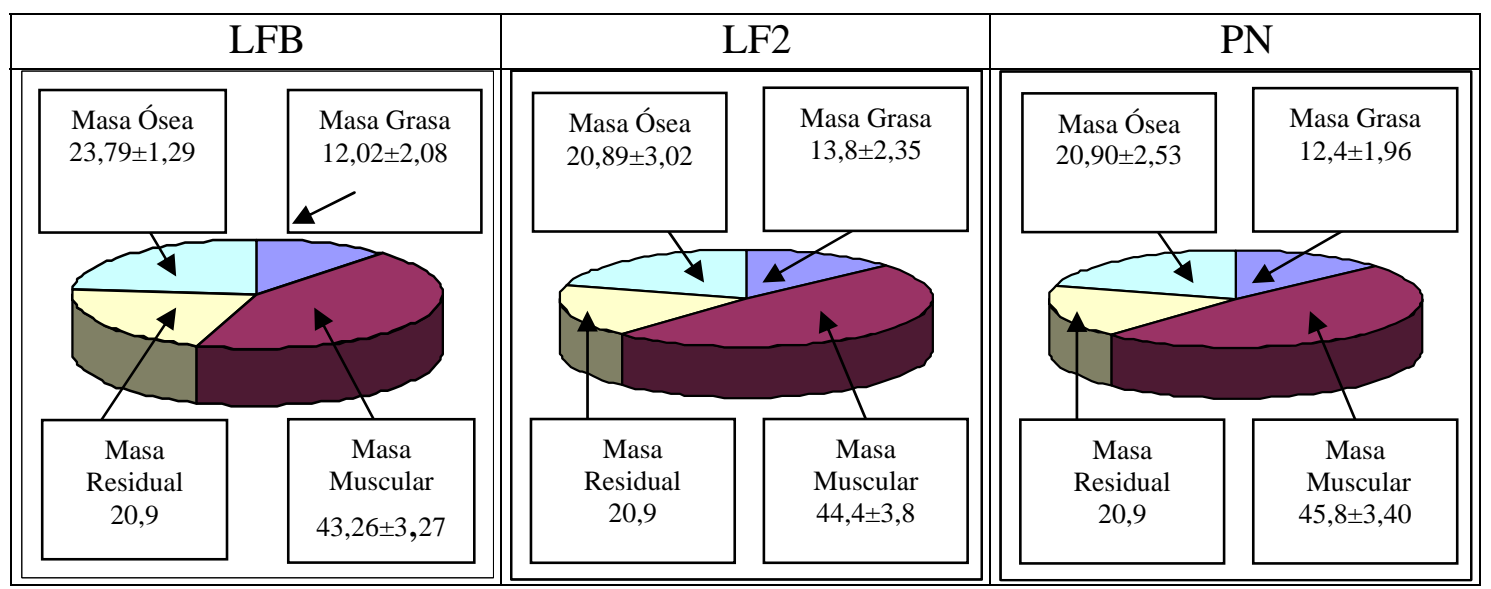

Figura 2: Distribución (\%) de la composición corporal en función de la categoría.

En la tabla II y la figura 3 se muestran los valores (media \pm D.E) obtenidos para los tres componentes del somatotipo en las tres categorías. Las jugadoras de LF2 presentan el componente endomórfico más alto; con lo que respecta al componente mesomórfico, son las 
Salgado, I.; Sedano, S.; de Benito, A.; Izquierdo, J.M.; Cuadrado, G. (2009). Perfil antropométrico de las jugadoras de baloncesto españolas. Análisis en función del nivel competitivo y de la posición específica de juego. Revista Internacional de Ciencias del Deporte. 15(5), 1-16. http://www.cafyd.com/REVISTA/01501.pdf

jugadoras de LFB las que presentan valores más elevados; por último, son las jugadoras de LFB las que presentan un mayor componente ectomórfico. Se encontraron diferencias estadísticamente significativas en el componente mesomórfico entre LFB y PN ( $\mathrm{p}<0.026)$, y en el componente ectomórfico entre LFB y LF2 $(\mathrm{p}<0,006)$.

Tabla II: Resultados obtenidos en los componentes del somatotipo para todas las categorías.

\begin{tabular}{|c|c|c|c|c|c|}
\hline Variables & LFB & LF2 & PN & F & $p$ \\
\hline Endomorfia & $3,67 \pm 1,02$ & $4,33 \pm 0,97$ & $4,14 \pm 0,84$ & 2.431 & .101 \\
\hline Mesomorfia & $3,35 \pm 1,64_{\mathrm{a}}$ & $2,77 \pm 1,82_{\mathrm{ab}}$ & $1,81 \pm 0,96_{\mathrm{b}}$ & 17.822 & .025 \\
\hline Ectomorfia & $3,36 \pm 0,84_{\mathrm{a}}$ & $2,22 \pm 1,06_{\mathrm{b}}$ & $2,51 \pm 0,84_{\mathrm{ab}}$ & 24.055 & .012 \\
\hline
\end{tabular}

Nota: Los datos en la misma fila, con distinto subíndice, muestran diferencias estadísticamente significativas, $(\mathrm{p}<0.05)$.

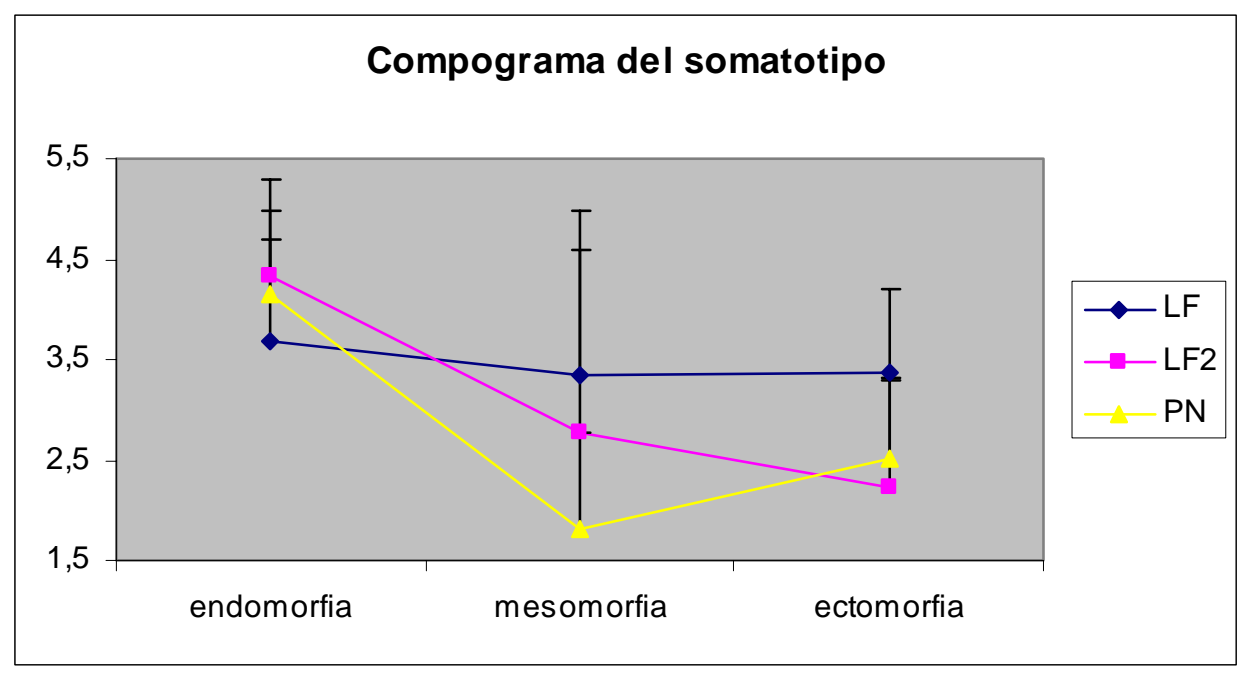

Figura 3. Componentes del somatotipo en función de la categoría.

En cuanto al somatotipo, las jugadoras de LFB tienen una tendencia al somatotipo central, mientras que las jugadoras de LF2 se sitúan en la zona del somatotipo meso-endomorfo y las de PN en la del ecto-endomorfo (Figura 4). 
Salgado, I.; Sedano, S.; de Benito, A.; Izquierdo, J.M.; Cuadrado, G. (2009). Perfil antropométrico de las jugadoras de baloncesto españolas. Análisis en función del nivel competitivo y de la posición específica de juego. Revista Internacional de Ciencias del Deporte. 15(5), 1-16. http://www.cafyd.com/REVISTA/01501.pdf

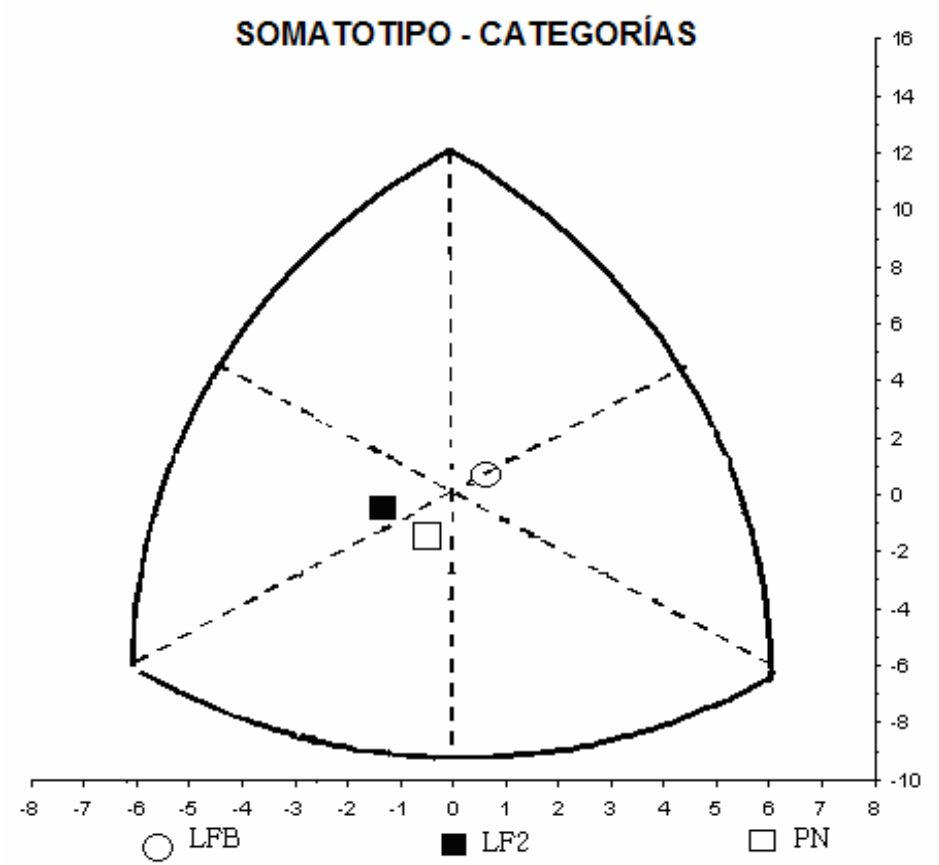

Figura 4. Somatocarta en función de la categoría.

Resultados obtenidos en función de la posición específica de juego

En la Tabla III se muestran los valores (media \pm D.E) para la masa corporal, la talla y la composición corporal en función de la posición específica de juego: bases (B), aleros (A), pívots (P). Al igual que ocurría en el análisis por nivel competitivo, los datos relativos al porcentaje de masa residual se han omitido, por ser idénticos, en términos de porcentaje, en todos los grupos analizados.

Tabla III: Resultados obtenidos en todas las variables, para todas las posiciones.

\begin{tabular}{|l|c|c|c|c|c|}
\hline \multicolumn{1}{|c|}{ Variable } & $\mathrm{B}$ & $\mathrm{A}$ & $\mathrm{P}$ & $\mathrm{F}$ & $\mathrm{P}$ \\
\hline Masa Corporal (Kg) & $64,7 \pm 4,3$ & $70,5 \pm 7,2$ & $79,6 \pm 9,8$ & 14.067 & .000 \\
\hline Talla (cm) & $171,2 \pm 4,3$ & $176,6 \pm 6,9$ & $184,1 \pm 6,9$ & 15.567 & .000 \\
\hline Porcentaje de grasa & $11,8 \pm 1,8$ & $12,9 \pm 1,9$ & $13,4 \pm 2,7$ & 1.688 & .195 \\
\hline $\begin{array}{l}\text { Porcentaje de masa } \\
\text { muscular }\end{array}$ & $46,1 \pm 4,7$ & $44,6 \pm 4,7$ & $44,2 \pm 4,2$ & 1.283 & .286 \\
\hline $\begin{array}{l}\text { Porcentaje de masa } \\
\text { ósea }\end{array}$ & $21,2 \pm 1,3$ & $21,6 \pm 1,4$ & $21,5 \pm 1,4$ & 0.256 & .775 \\
\hline
\end{tabular}

Se apreciaron diferencias estadísticamente significativas en el caso de la masa corporal y la talla. En ambos casos esas diferencias se encontraron entre B y $\mathrm{P}$ y A y $\mathrm{P}$, siendo $\mathrm{P}$ las jugadoras más altas y más pesadas (Figura 5). 


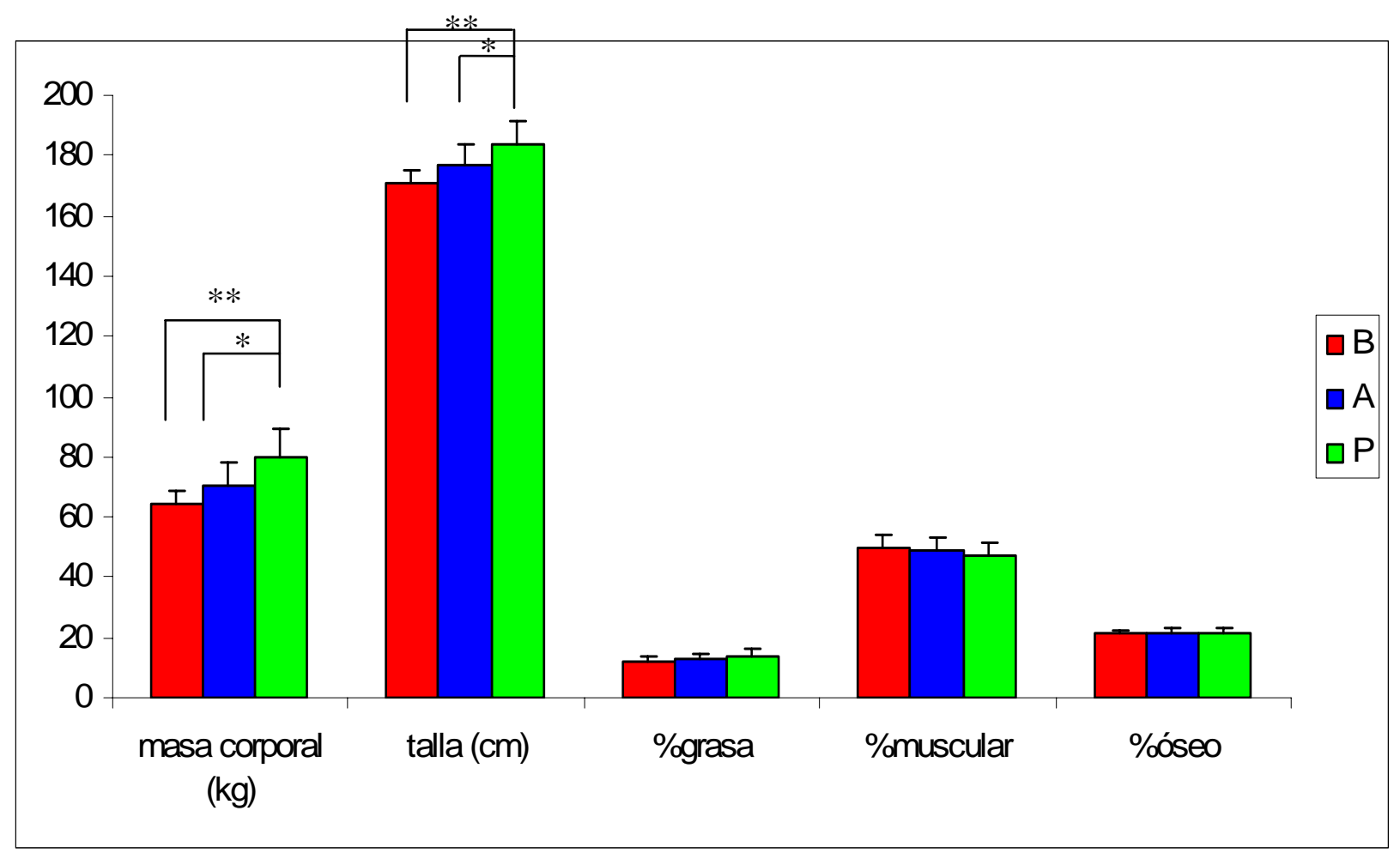

Figura 5. Relación de las diferencias significativas entre posiciones. ${ }^{*} \mathrm{p}<0,05$; ${ }^{* *} \mathrm{p}<0,01$. MC masa corporal; $\mathrm{T}$ talla; G graso; M muscular; O óseo; R residual.

Aunque el ANOVA no revela la existencia de diferencias estadísticamente significativas en la composición corporal, hay que citar que B presenta valores más bajos con respecto al porcentaje de grasa. Con el porcentaje muscular, ocurre a la inversa, siendo $\mathrm{P}$ las que presentan un porcentaje menor. Por último, con respecto al porcentaje óseo, B muestra los valores más bajos.

En la tabla IV y la figura 6 se muestran los valores (media \pm D.E) obtenidos para cada componente del somatitopo en las tres posiciones de juego. Se observa que P obtiene los mayores valores en el componente endomórfico y ectomórfico, mientras que en el componente mesomórfico B obtienen los valores más altos. No obstante, no se aprecian diferencias significativas entre los tres grupos para ninguno de los componentes.

Tabla IV: Resultados obtenidos en los componentes del somatotipo para todas las posiciones.

\begin{tabular}{|c|c|c|c|c|c|}
\hline Variables & $\mathrm{B}$ & $\mathrm{A}$ & $\mathrm{P}$ & $\mathrm{F}$ & $\mathrm{p}$ \\
\hline Endomorfia & $3,86 \pm 0,97$ & $4,17 \pm 0,74$ & $4,18 \pm 1,13$ & 5.179 & .342 \\
\hline Mesomorfia & $2,87 \pm 1,96$ & $2,50 \pm 1,61$ & $2,32 \pm 1,43$ & 4.321 & .569 \\
\hline Ectomorfia & $2,28 \pm 1,22$ & $2,58 \pm 0,65$ & $2,66 \pm 1,20$ & 4.378 & .625 \\
\hline
\end{tabular}

Nota: Los datos en la misma fila, con distinto subíndice, muestran diferencias estadísticamente significativas, $(\mathrm{p}<0.05)$. 


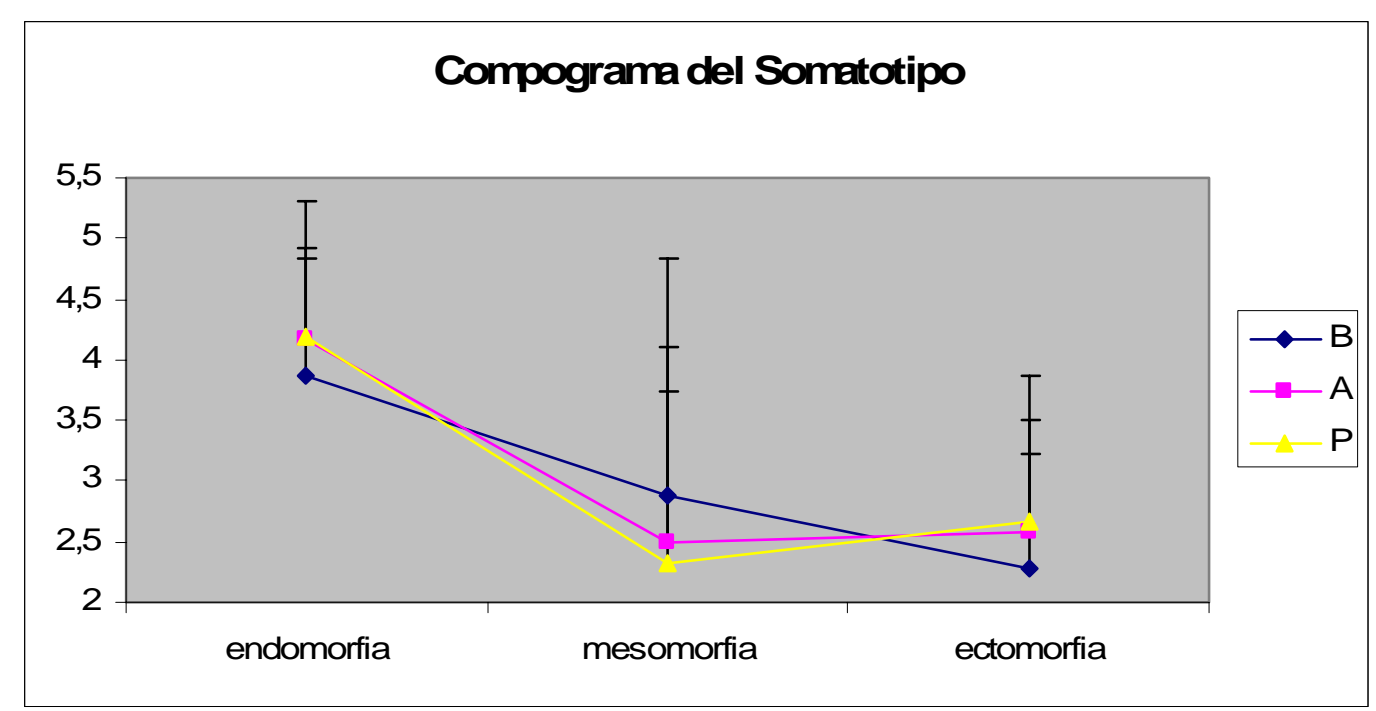

Figura 6. Componentes del somatotipo en función del puesto específico de juego.

Al analizar la somatocarta de las jugadoras en función de la posición, se observa la existencia de diferencias entre unas y otras (Figura 7). A pesar de que en los tres grupos predomina el componente endomórfico, en dos de ellos, A y P, la ectomorfia es mayor que la mesomorfia, por lo que dichos grupos se encuadran dentro del somatotipo denominado como ectoendomorfo. A pesar de que ambos grupos se hallan dentro de un mismo somatotipo resulta evidente que existe una diferencia entre ellos, diferencia que se ha cuantificado calculando la distancia de dispersión de los somatotipos medios (SDD del SM). En este caso la SDD del SM es superior a 2 por lo que, siguiendo a Hebbelink (citado por Esparza y cols., 1993), la distancia entre ambos somatotipos es estadísticamente significativa.

Por otro lado, el grupo de B se ubica dentro del somatotipo denominado como endomorfobalanceado, lo que viene a significar que también la endomorfia es dominante, pero en este caso la mesomorfia y la ectomorfia son similares, (se diferencian en menos de medio punto).

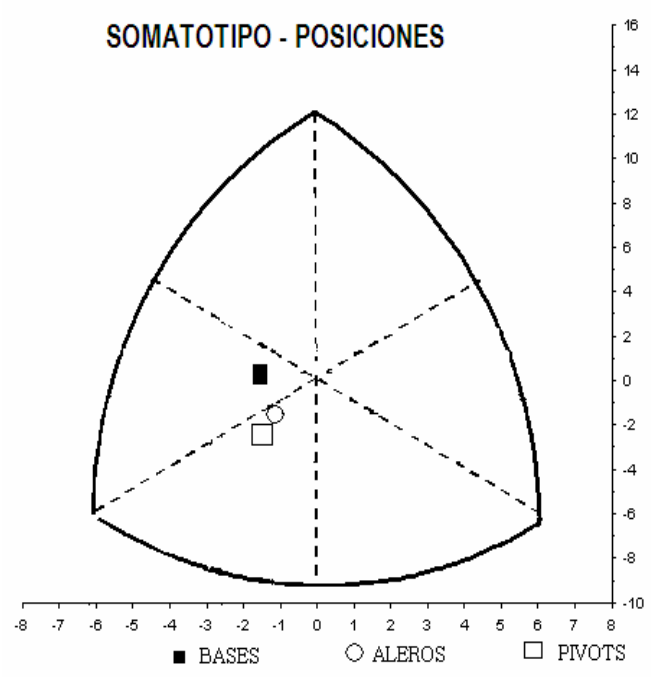

Figura 7: Somatocarta en función del puesto específico de juego. 
Salgado, I.; Sedano, S.; de Benito, A.; Izquierdo, J.M.; Cuadrado, G. (2009). Perfil antropométrico de las jugadoras de baloncesto españolas. Análisis en función del nivel competitivo y de la posición específica de juego. Revista Internacional de Ciencias del Deporte. 15(5), 1-16. http://www.cafyd.com/REVISTA/01501.pdf

\section{Discusión}

$\mathrm{Al}$ analizar los resultados lo primero que se observa es que la variable masa corporal no se relaciona de manera directa con el nivel competitivo ya que las jugadoras de LF2 son las que obtienen mayores registros en masa corporal, seguidas por las de LFB y por último las de PN. No obstante, en la variable talla sí se observa esta relación directa puesto que, a mayor categoría, se alcanzan mayores registros, siendo las jugadoras de LFB las más altas, seguidas por las de LF2 y PN respectivamente.

Withers (1987) obtuvo unos valores en la masa corporal de 68,0 $\pm 8,2 \mathrm{~kg}$, resultado que se encuentra muy cercano al del presente estudio para el grupo de $\mathrm{PN}$, pero que es considerablemente inferior al del grupo de LFB y LF2. Lo mismo ocurre con otros estudios (Bale, 1991; Häkkinen, 1993; Rodríguez-Alonso, 1998), donde los registros obtenidos son inferiores a los de este trabajo, siendo el grupo de PN el que más se aproxima a esos valores. Los resultados más cercanos a los aquí reseñados son los de Ackland y cols., (1997) quienes al medir la masa corporal de las participantes en el Campeonato del Mundo de Baloncesto en Australia encontraron unos valores medios de $74,0 \mathrm{~kg}$. También se encuentran próximos los resultados de Rodríguez-Alonso (2003) y Bayios y cols. (2006), que aunque superan los valores registrados en el grupo de PN, también son inferiores a los de los grupos de LFB y LF2.

En la muestra estudiada se encontraron diferencias estadísticamente significativas en la masa corporal entre niveles, algo que no ocurrió en el estudio de Rodríguez-Alonso (2003) al comparar entre el Equipo Nacional Olímpico $(71,7 \pm 7,6 \mathrm{~kg})$ y un equipo de la Primera División de la Liga Española (71,9 \pm 8,7kg). Por otro lado, los registros obtenidos en estudios efectuados en baloncesto masculino tampoco muestran diferencias estadísticamente significativas en función del nivel (Sallet y cols. 2005). Según lo señalado, aunque aparentemente los resultados revelan que la masa corporal tiene relación con el rendimiento en baloncesto, ésta afirmación no puede efectuarse de manera categórica, si se comparan los datos obtenidos en la presente muestra con estudios efectuados con anterioridad.

Con respecto a la variable talla los resultados obtenidos en el presente estudio están por encima de los registrados por otros autores. Únicamente el grupo de PN se acerca a dichos valores. Los valores obtenidos por el grupo de LF2 están en consonancia con el estudio de Rodríguez-Alonso (2003) que obtuvo una media de 180,9 $\pm 8,0 \mathrm{~cm}$. No obstante, los presentes resultados concuerdan con los de varios estudios realizados con atletas de equipos nacionales donde los valores de la talla oscilaban entre 176 y 181,8 cm, (Spurgeon y cols., 1981; Piechaczek, 1990; Ackland y cols., 1997; Monyeki y cols., 1998), siendo únicamente el grupo de $\mathrm{PN}$ el que quedaría por debajo de estos rangos. Los resultados están en línea con los de otros autores al afirmar que a medida que aumenta la categoría, aumenta la talla (Spurgeon y cols., 1980; Rodríguez-Alonso, 1998; Rodríguez-Alonso, 2003; Bayios y cols., 2006). En baloncesto masculino esta tendencia ha sido observada por numerosos autores (Sodhi, 1980; Toriala, 1987; Sallet y cols, 2005). Por tanto, y como afirman otros estudios (Alexander, 1976; Fleck y cols., 1985; Carter y cols., 1990) tanto la talla en sí misma como la influencia positiva que ésta tiene sobre la longitud de todos los segmentos corporales aparentemente afecta positivamente al rendimiento del jugador de baloncesto. 
Salgado, I.; Sedano, S.; de Benito, A.; Izquierdo, J.M.; Cuadrado, G. (2009). Perfil antropométrico de las jugadoras de baloncesto españolas. Análisis en función del nivel competitivo y de la posición específica de juego. Revista Internacional de Ciencias del Deporte. 15(5), 1-16. http://www.cafyd.com/REVISTA/01501.pdf

En lo que a composición corporal se refiere, nos encontramos ante una distribución diferente de los tejidos corporales en las distintas categorías. Así, si bien se aprecia que el grupo de LFB es el que menor porcentaje de grasa obtiene, seguido por el de PN y el de LF2, cabe destacar que el porcentaje de masa muscular más elevado pertenezca al grupo de PN, seguido por el de LF2 y por último el de LFB. Este dato llama la atención, puesto que el volumen de entrenamiento y de competiciones es mayor para los grupos de LFB y LF2 con respecto al de PN, y aunque ese volumen de entrenamiento parece ejercer un efecto positivo sobre el porcentaje de grasa, disminuyéndolo, no ocurre así con el porcentaje muscular. Esa ausencia de relación entre la masa muscular y el rendimiento puede deberse a una limitación en el cálculo de la masa muscular mediante la fórmula de Matiegka, más habitual en sujetos sedentarios que en deportistas, y el efecto que ejerce el cálculo de la masa residual sobre la masa muscular. Así podría ocurrir que, el aumento de la masa muscular de un sujeto como consecuencia del entrenamiento se pudiera ver enmascarado por la forma de calcular la masa residual (una constante por la masa corporal).Por último, con respecto al porcentaje de masa ósea, es el grupo de LFB el que presenta mayores valores, mientras que el grupo de LF2 y el de PN presentan resultados similares.

Los valores de porcentaje de grasa aquí registrados se encuentran por debajo de la cifra que Bale (1991) indicaba como normal para jugadoras de baloncesto, (18\%). Si comparamos los datos obtenidos con trabajos de Häkkinen (1993) y Bayios y cols. (2006) observamos que también estos autores registraron valores más elevados en lo que a porcentaje de grasa se refiere. No ocurre así en el estudio de Gualdi-Russo y cols. (1992), donde se obtivieron unos valores de 13,88 $\pm 2,97$ para las jugadoras de la Primera División y de 14,15 $\pm 3,61 \%$ para las jugadoras de Segunda División, aproximándose más a los valores obtenidos en la muestra aquí estudiada. Lo mismo ocurre con el trabajo de Fernández-Río (2000) donde se obtuvo un valor de 14,3 $\pm 2,9$ para un grupo de jugadoras de LF2. Lo que queda de manifiesto con esos estudios es que a medida que aumenta la categoría, disminuye el porcentaje de grasa de las jugadoras (Gualdi-Russo y cols., 1992; Sallet y cols., 2003; Bayios y cols., 2006) aspecto que en el presente estudio se produce sólo parcialmente, puesto que aunque las jugadoras de LFB son las que menor porcentaje de grasa presentan, esa relación categoría-porcentaje de grasa no es lineal, debido a que el grupo de LF2 presenta mayores porcentajes que el grupo de LFB y de PN, rompiéndose dicha relación.

Con lo que respecta al porcentaje de masa muscular, si bien Casajús y Aragonés (1997) señalaban que la principal diferencia en variables antropométricas en función del nivel competitivo radicaba en un mayor desarrollo muscular de los deportistas de mayor nivel, esto no ocurre en la presente muestra, donde son las jugadoras de menor nivel las que obtienen mayores registros, seguidas de las del nivel medio y en último lugar las de mayor nivel. Esparza y cols. (1993), al hablar de la composición corporal de un grupo de mujeres atletas sitúa los valores del porcentaje de masa muscular entre el 50\% y 52\%, valores que sí encontraríamos en el grupo de $\mathrm{PN}$, pero que quedarían un poco por encima de los grupos de LF2 y LFB. A diferencia de lo que ocurre en el presente trabajo, otros autores que han analizado grupos de mujeres practicantes de deportes colectivos señalan que a medida que aumenta el nivel competitivo aumenta de manera significativa el porcentaje de masa muscular.

El somatotipo permite valorar la morfología del cuerpo y en este caso los resultados obtenidos revelan que las jugadoras de LF2 presentan el componente endomórfico más alto, seguidas de las de PN y las de LFB. Con lo que respecta al componente mesomórfico, son las jugadoras de LFB las que presentan valores más elevados, seguidas de las jugadoras de LF2 y PN 
Salgado, I.; Sedano, S.; de Benito, A.; Izquierdo, J.M.; Cuadrado, G. (2009). Perfil antropométrico de las jugadoras de baloncesto españolas. Análisis en función del nivel competitivo y de la posición específica de juego. Revista Internacional de Ciencias del Deporte. 15(5), 1-16. http://www.cafyd.com/REVISTA/01501.pdf

respectivamente. Por último, son las jugadoras de LFB las que presentan un mayor componente ectomórfico, seguidas de las de PN y por último las de LF2. No se encuentran diferencias estadísticamente significativas en el componente endomórfico, pero sí existen en el componente mesomórfico entre LFB y PN, y en el componente ectomórfico entre LFB y PN. Según Esparza y cols., (1993) los deportistas muestran tendencias marcadas al predominio del segundo componente del somatotipo (mesomorfia) algo que en el presente estudio no ocurre en ninguno de los niveles competitivos analizados.

Con respecto a los valores obtenidos en el componente endomórfico, el grupo de LFB coincide con la mayoría de estudios realizados, que sitúan dicho componente entre unos valores de entre 3.4 y 3.9. No obstante, nuestro grupo de LF2 y de PN obtiene valores superiores y únicamente coincide con los registros obtenidos por Heath (citado por Carter, 1970), pero que al ubicarse en 1970 nos puede hacer ver que desde entonces ha existido un cambio en la tendencia del componente endomórfico hacia valores menos elevados. Por otro lado, con lo que respecta al componente mesomórfico, ocurre algo parecido ya que el grupo de LFB se encuentra dentro de los valores propuestos por los estudios más actuales, mientras que los resultados registrados por los grupos de LF2 y PN están más alejados de los mismos. Y por último, en referencia al valor ectomórfico, el grupo de LFB es esta vez quien obtiene datos muy superiores a los de los estudios citados anteriormente, mientras que los grupos de LF2 y PN se encuentran próximos a los mismos.

En lo que hace referencia a la relación existente entre los valores del somatotipo y el nivel competitivo, en la muestra analizada se observa que a medida que disminuye el nivel aumenta el valor de endomorfia (aunque cabe resaltar que el grupo de LF2 presenta un valor mayor que el de PN) y disminuye la mesomorfia y la ectomorfia. Este resultado está en línea con diversos estudios (Gualdi-Russo y cols., 1993; Bayios y cols., 2006). Sabiendo que la endomorfia se relaciona con el porcentaje de grasa, este valor estaría en consonancia con los datos señalados anteriormente para el porcentaje de grasa algo que no ocurre con los valores del componte mesomórfico, que teóricamente deberían relacionarse con el porcentaje de músculo y de hueso. Sedano y cols. (en prensa) se encuentran con un caso similar cuando analizan una muestra de mujeres futbolistas de diferentes niveles competitivos. Por último, en el componente ectomórfico, que se refiere al predominio de medidas longitudinales sobre transversales, son las jugadoras de LFB las que obtienen valores más elevados, datos que concuerdan con los valores obtenidos en los parámetros que medían la relativa linealidad de las jugadoras. Por otro lado, si bien la mayoría de estudios sobre el somatotipo sitúan a las jugadoras de baloncesto dentro del somatotipo denominado como meso-endomorfo (Carter, 1981; Eiben, 1981; Carter y cols., 1990; Monyeki y cols., 1998; Bayios y cols., 2006), esto solo ocurre en el presente estudio en el caso del grupo de LF2.

En lo que hace referencia a los resultados obtenidos en función de la posición específica de juego, en las variables masa corporal y talla, $\mathrm{P}$ son las más pesadas y más altas, seguidas por A y por último B, lo que está en consonancia con otros estudios realizados en baloncesto tanto femenino (Bale, 1991; Canda (citado por Esparza y cols., 1993); Terrados y cols., 1995; Fernández-Río, 2000) como masculino (Sodhi, 1980; Toriola y cols., 1985; Galiano, 1987; Tocigl, 1991; Dospaj y Milisic, 1993; Janeira, 1994; Latin y cols., 1994; Berg y Latin, 1995; Jordane y cols., 1999; Apostolidis y cols., 2003; Sallet y cols., 2005).

El análisis de la composición corporal revela que $\mathrm{P}$ son las que tienen un porcentaje de grasa superior, siendo $\mathrm{B}$ las que menor porcentaje de grasa obtienen y situándose en un punto intermedio A. En el caso del porcentaje muscular ocurre a la inversa, ya que B tiene una 
Salgado, I.; Sedano, S.; de Benito, A.; Izquierdo, J.M.; Cuadrado, G. (2009). Perfil antropométrico de las jugadoras de baloncesto españolas. Análisis en función del nivel competitivo y de la posición específica de juego. Revista Internacional de Ciencias del Deporte. 15(5), 1-16. http://www.cafyd.com/REVISTA/01501.pdf

mayor masa muscular, seguidas por A y finalmente P. Estos resultados coinciden con los de otros estudios de baloncesto femenino (Riezebos y cols., 1983; Bale, 1991; Smith y cols., 1991; Häkkinen, 1993; Rodríguez-Río, 2000).

En lo que hace referencia al somatotipo, $\mathrm{P}$ presentan un mayor valor en endomorfia y ectomorfia, y B poseen valores superiores de mesomorfia, aunque en ninguno de los casos aparecen diferencias estadísticamente significativas entre posiciones. En el estudio efectuado por Canda (citado por Esparza y cols., 1993), también P obtienen un mayor valor de endomorfia, aunque no ocurre así con el valor de ectomorfia, siendo A las que obtienen resultados más elevados. Con lo que respecta al componente de mesomorfia también en su estudio B son las que obtienen mayores registros. Por otro lado, el estudio de Bale (1991) también muestra que $\mathrm{P}$ obtienen mayores valores en el componente de endomorfia, pero sus resultados variaban con respecto a los del presente estudio en los componentes de mesomorfia y ectomorfia, siendo A las que obtenían mayores valores en ambos.

Galiano (1987), en un trabajo cuyo propósito era estudiar los diversos somatotipos en jugadores de baloncesto, observó que estos presentan un equilibrio entre los diferentes componentes primarios que componen la morfología humana, siendo el somatotipo predominante en $\mathrm{B}$ el ecto-mesomórfico. Esto no ocurre aquí, donde $\mathrm{B}$ presentan un somatotipo denominado como endomorfo-balanceado. En ese mismo estudio, Galiano establece el somatotipo de A en ecto-mesomórfico en contraste con el endo-ectomórfico que caracteriza a P. Esto difiere con los presentes resultados, donde A y P obtenían un somatotipo del tipo ecto-endomorfo. Pos su parte, Tocigl (1991), realizó un estudio consistente en asociar la estructura factorial de las variables morfológicas a la estructura factorial de las variables psicomotrices, permitiendo identificar cinco perfiles de jugadores. Concretamente, a B los agrupa dentro del perfil 3 , en los que se incluyen los de tipo mesomorfo, resultado que no coincide con el de la presente muestra. No obstante, este autor agrupa a A dentro del perfil 4, que abarca a los jugadores de tipo ecto-endomorfo, lo que sí coincide con la muestra de A. Por último, él engloba a $\mathrm{P}$ dentro del perfil 5, correspondiente a aquellos de tipo ectomorfo, mientras que en la muestra aquí estudiada, $\mathrm{P}$ se agrupan en el somatotipo denominado como ecto-endomorfo.

\section{Conclusiones}

1. Se encontraron diferencias significativas en la mayoría de las variables antropométricas, de composición corporal y de los componentes del somatotipo estudiados en función del nivel competitivo. No obstante, estas diferencias no fueron favorables siempre al grupo de mayor nivel, lo que sugiere que posiblemente dichas variables por sí solas no sean los factores más determinantes para el rendimiento en baloncesto, aunque sí que han de considerarse importantes para el mismo.

2. Podemos hablar de la existencia de un perfil cineantropométrico específico en función de la posición de juego. B son las que tienen menores valores en la talla, la masa corporal y el porcentaje de grasa, mientras que presentan los valores más altos en cuanto al porcentaje de masa muscular. En una situación intermedia estarían A. Y por último P, que eran las más altas, las más pesadas, y las que presentan un mayor valor de porcentaje de grasa, y el menor valor en cuanto al porcentaje muscular. Dicho perfil cineantropométrico estaría en relación con las posiciones ocupadas en el campo y las funciones desarrolladas en las mismas. 
Salgado, I.; Sedano, S.; de Benito, A.; Izquierdo, J.M.; Cuadrado, G. (2009). Perfil antropométrico de las jugadoras de baloncesto españolas. Análisis en función del nivel competitivo y de la posición específica de juego. Revista Internacional de Ciencias del Deporte. 15(5), 1-16. http://www.cafyd.com/REVISTA/01501.pdf

\section{Referencias Bibliográficas}

Ackland, R.T.; Schreiner, B.A. y Kerr, A. D. (1997). Absolute size and proportionality characteristics of World Championship female basketball players. Journal of Sports Sciences, 15 (5), 485-490.

Alexander, M.J. (1976). The relationship of somatotype and selected anthropometric measures to basketball performance in highly skilled females. Research quarterly for exercise and sport, 47, 575-585.

Apostolidis, N.; Nazis, G.P.; Bolatoclou, T.; y Geladas, N.D. (2003). Physiological and technical characteristics of elite young basketball players. The Journal of Sports Medicine and Physical Fitness, 44 (2), 157-163.

Bale, P. (1991). Anthropometric, body composition and performance variables of young elite female basketball players.The Journal of Sports Medicine and Physical Fitness, 31 (2), 173-177.

Bayios, I.A.; Bergeles, N.K.; Apostolidis, N.G.; Noutsos, K.S. y Koskolou, M.D. (2006). Anthropometric, body composition and somatotype differences of Greek elite female basketball, volleyball and handball players. The Journal of Sports Medicine and Physical Fitness, 46 (2), 271-280.

Berg, K. y Latin, R. (1995). Comparison of physical and performance characteristics of NCAA Division I Basketball and Football Players. The Journal of Strength and Conditioning Research, 8 (1), 22-26.

Carter, J.E.L. (1970). The somatotype of athletes: A review. Human Biology, 42, 535569.

Carter, J.E.L. (1975). The Heath-Carter somatotype method. San Diego: San Diego State University.

Carter, J.E.L. (1981). Somatotypes of female athletes. En: Borms, J., Hebbelinck, M., Venerando, A., editors. The female athlete. Basel: S Karger Press; 15: 85-116.

Carter, J.E.L. y Heath, B.H. (1990). Analysis. En: Lasker, G.W., Mascie-Taylor, C.G.N y Roberts, D.F (Eds). Somatotyping. Development and applications. pp. 398-420. Cambridge: Cambridge University Press.

Casajaús, J.A. y Aragonés, M.T. (1997). Estudio cineantropométrico del futbolista profesional español. Archivos de Medicina del Deporte, 14 (59), 177 - 184.

Cometti, G. (2002). La preparación física en el baloncesto. Barcelona: Paidotribo.

Dospaj, M.; Milisic, B. (1993). Vaznot metode modelavanja u procesu sportske pripreme u kosarci. Jugoslovenski zavod za fizicku kulturu i medicinu sporta. Yugoslavia.

Eiben, O. (1981). Physique of female athletes: anthropological and proporcional análisis. En: Borms, J., Hebbelinck, M., Venerando, A., editors. The female athlete. Basel: S Karger Press; 15: 127-141.

Esparza, F.; Alvero, J.R.; Aragonés, M.T.; Cabañas, M.D.; Canda, A.; Casajaús, J.A.; Chamorro, M; Galiano, D.; González, J.M.; Pacheco, J.L.; Porta, J.; Rodríguez, F.; Tejedo, A. (1993). Manual de Cineantropometría. Navarra: FEMEDE.

Faulkner, J.A. (1968). Physiology of swimming and diving. En: Falls, H. Exercise Physiology. Baltimore, Academic Press.

Fernández-Río, J.; Rodríguez, M.; Terrados, N.; Pérez-Landaluce, J. (2000). Valoración fisiológica en jugadoras de baloncesto. Apunts. Medicina de L'esport, 132, 11-17. 
Salgado, I.; Sedano, S.; de Benito, A.; Izquierdo, J.M.; Cuadrado, G. (2009). Perfil antropométrico de las jugadoras de baloncesto españolas. Análisis en función del nivel competitivo y de la posición específica de juego. Revista Internacional de Ciencias del Deporte. 15(5), 1-16. http://www.cafyd.com/REVISTA/01501.pdf

Fleck, J.S.; Case, S.; Puhl, J.; Handle, V.P. (1985). Physical and physiological characteristics of elite women volleyball players. The Canadian Journal of Sports Sciences, 10, 122-126.

Franco Bonafonte, L. (1998). Physiology of basketball . Archivos de Medicina del Deporte, Volumen XV. № 68: 471-477.

Galiano, D. (1987). Características del jugador. Apunts. Medicina de L'esport, 7, 93-98.

Garrido, R.P.; González, M.; Félix, A.; Pérez, J. (2004). Composición corporal de los futbolistas de equipos alicantinos. Selección, 13(4), 155 - 163.

Gualdi-Russo, E.; Gruppioni, G.; Gueresi, P.; Belcastro, G.M.; Marchesini, V. (1992). Skinfolds and body composition of sports participants. The Journal of Sports Medicine and Physical Fitness, 32, 303-313.

Gualdi-Russo, E.; Graziani, I. (1993). Anthropometric somatotype of Italian sport participants. The J ournal of Sports Medicine and Physical Fitness, 33, 281-291.

Häkkinen, K. (1993). Changes in physical fitness profile in female basketball players during the competitive season including explosive type strength training. The Journal of Sports Medicine and Physical Fitness, 33, 19-26.

Janeira, M.A.; Maia, J.A. (1995). Estudo diferencial em basquetebolistas seniores masculinos: Uma abordagem multivariada. 4ํㅡㄹ Congreso de Educaçao Física. Coimbra. Portugal.

Jordane, F.; Martin, J. (1991). Baloncesto. Bases para el alto rendimiento. Barcelona: Hispano Europea.

Latin, R.W.; Berg, K.; Baechle, T. (1994). Physical and performance characteristics of NCAA division. I male basketball players. The Journal of Strength and Conditioning Research, 8, 214-218.

Liparotti, J.R. (2004). Aplicaciones prácticas de datos de composición corporal en futbolistas universitarios brasileños. Training fútbol, (100), 36 - 43.

Lorenzo, A. (1998). Adecuación de la preparación física en el entrenamiento técnicotáctico en baloncesto. Educación física y deportes. http://www. efdeportes.com/.

Matiegka, J. (1921). The testing of physical efficiency. The American Journal of Physical Anthropology, 4, 223-230.

Meszaros, J.; Mohasci, J. (1982). An anthropometric study of top level athletes in view of the changes that took place in the style of some ball games. Humabilogia Budapestinensis, 13, 15-20.

Monyeki, K. D.; Amusa, L. O.; Toriola, A. L.; Wekesa, M.; Ridder, J. H. (1998). Somatotypes of elite basketball and handball players at the $6^{\text {th }}$ all Africa games. The African J ournal of Physical Education, 4, 1-7.

Piechaczek, H. (1990). Body structure of male and female basketball players. Biology of Sports, 7, 273-285.

Riezebos, L. M.; Paterson, H. D.; Hall, R. C.; Yuhasz, S. M. (1983). Relationship of selected variables to performance in women's basketball. The Canadian Journal of Sports Sciences, 8, 34-40.

Rodríguez-Alonso, M.; Terrados, N.; Pérez-Landaluce, J.; Fernández, B.; García-Herrero, F. (1998). Déficit máximo acumulado de oxígeno en baloncesto femenino. Archivos de Medicina del Deporte XV (64), 115-122. 
Salgado, I.; Sedano, S.; de Benito, A.; Izquierdo, J.M.; Cuadrado, G. (2009). Perfil antropométrico de las jugadoras de baloncesto españolas. Análisis en función del nivel competitivo y de la posición específica de juego. Revista Internacional de Ciencias del Deporte. 15(5), 1-16. http://www.cafyd.com/REVISTA/01501.pdf

Rodríguez-Alonso, M.; Fernández-García, J.; Pérez-Landaluce, J.; Terrados, N. (2003). Blood lactate and heart rate during nacional and internacional women's basketball. The J ournal of Sports Medicine and Physical Fitness, 43, 432-436.

Sallet, P.; Perrier, D.; Ferret, J. M.; Vitelli, V.; Baverel, G. (2005). Physiological differences in professional basketball players as a function of playing position and level of play. The J ournal of Sports Medicine and Physical Fitness, 45, 291-294.

Smith, H.; Thomas, S. (1991). Physiological characteristics of elite female basketball players. Canadian Journal of Applied Sports Sciences, 16 (4), 289-295.

Sodhi, H. S. (1980). Kinanthropometry and performance of top ranking indian basketball players. British J ournal of Sports Medicine, 14 (2,3), 139-144.

Spurgeon, H. J.; Spurgeon, L. N.; Giese, K. W. (1980). Physique of world class female basketball players. Scandinavian J ournal of Medicine and Science in Sports, 2, 63-69.

Spurgeon, H. J.; Spurgeon, L. N.; Giese, K. W. (1981). Measures of body size and formo $f$ elite female basketball players. En: Borms J, Hebbelinck $M$, Venerando A, editors. The female athlete. Basel: S Karger Press, 15, 192-200.

Terrados, N.; Fernández, B.; Pérez-Landaluce, J.; Rodríguez, M.; Coloma, M.; Buceta, J. M. (1995). Physiological aspects of woman's baskeball. Medicine and Science in Sports and Exercise, 24, 142.

Toriola, A. L.; Salokum, S. O.; Mathur, D. N. (1985). Somatotype characteristics of male sprinters, basketball, soccer and field hochey players. International Journal of Sports Medicine, 6, 344-346.

Würch, A. (1974). La femme et le sport. Mèdicine Sportive. París, 5 (1).

Zaragoza, J. (1996). Análisis de la actividad competitiva. Clínic, 34, 15-18. 